\title{
Calibration of spaceborne scatterometers using tropical rain forests
}

David G. Long

david_long@byu.edu

Gary B. Skouson

Follow this and additional works at: https://scholarsarchive.byu.edu/facpub

Part of the Electrical and Computer Engineering Commons

\section{Original Publication Citation}

Long, D. G., and G. B. Skouson. "Calibration of Spaceborne Scatterometers using Tropical Rain Forests." Geoscience and Remote Sensing, IEEE Transactions on 34.2 (1996): 413-24

\section{BYU ScholarsArchive Citation}

Long, David G. and Skouson, Gary B., "Calibration of spaceborne scatterometers using tropical rain forests" (1996). Faculty Publications. 681.

https://scholarsarchive.byu.edu/facpub/681

This Peer-Reviewed Article is brought to you for free and open access by BYU ScholarsArchive. It has been accepted for inclusion in Faculty Publications by an authorized administrator of BYU ScholarsArchive. For more information, please contact ellen_amatangelo@byu.edu. 


\title{
Calibration of Spaceborne Scatterometers Using Tropical Rain Forests
}

\author{
David G. Long, Member, IEEE, and Gary B. Skouson
}

\begin{abstract}
Wind scatterometers are radar systems designed specifically to measure the normalized radar backscatter coefficient $\left(\sigma^{\circ}\right)$ of the ocean's surface in order to determine the near-surface wind vector. Postlaunch calibration of a wind scatterometer can be performed with an extended-area natural target such as the Amazon tropical rain forest. Rain forests exhibit a remarkably high degree of homogeneity in their radar response over a very large area though some spatial and temporal variability exist. In this paper we present a simple technique for calibrating scatterometer data using tropical rain forests. Using a polynomial model for the rolloff of $\sigma^{\circ}$ with incidence angle, the technique determines gain corrections to ensure consistency between different antennas and processing channels. Corrections for the time varying instrument gain are made consistent with a seasonally fixed rain forest response; however, without ground stations or aircraft flights, it is difficult to uniquely distinguish between seasonal variations in the rain forest and slow variations of the system gain. Applying the corrections, the intrinsic variability of the $\sigma^{\circ}$ of the rain forest is estimated to be \pm 0.15 $\mathrm{dB}$, which is the limit of the accuracy of calibration using the rain forest. The technique is illustrated with Seasat scatterometer (SASS) data and applied to ERS-1 Active Microwave Instrument scatterometer (Escat) data. Gain corrections of up to several tenths of a decibel are estimated for SASS. Corrections for Escat data are found to be very small, suggesting that Escat data is well calibrated.
\end{abstract}

\section{INTRODUCTION}

$\mathbf{S}^{\mathrm{s}}$ PACEBORNE wind scatterometers are an important element in future remote sensing systems because of their proven ability to make all-weather measurements of vector winds over the ocean. Wind scatterometry is an indirect measurement technique. The direct measurement is the backscattered power from the surface. Using the parameters of the radar equation, the normalized radar backscatter coefficient $\left(\sigma^{\circ}\right)$ is computed from the power measurement. Then, given several measurements of $\sigma^{\circ}$ taken from different azimuth angles, the near-surface wind is inferred using a geophysical model function in a process known as wind retrieval. Uncertainties and calibration errors in the quantities used to compute the parameters of the radar equation produce errors and uncertainty in the $\sigma^{\circ}$ measurements. These, in turn, result in errors in the retrieved wind.

Precise calibration (on the order of a few tenths of a decibel) of the scatterometer $\sigma^{\circ}$ measurements is required to achieve the desired wind measurement accuracy of $\pm 2 \mathrm{~m} / \mathrm{s}$ and $\pm 20^{\circ}$

Manuscript received December 20, 1994.

The authors are with the Electrical and Computer Engineering Department, Brigham Young University, Provo, UT 84602 USA (e-mail: long@ee.byu.edu).

Publisher Item Identifier S 0196-2892(96)00992-8.
[7]. This level of precision in radar calibration is difficult to achieve using prelaunch calibration alone so extensive postlaunch calibration campaigns are planned for the period shortly after the launch of a new instrument. Traditionally, two key approaches to postlaunch calibration have been used: 1) ground stations (first used by Escat) and 2) homogeneous extended-area targets such as tropical rain forests. The latter approach was pioneered for SASS and has been used for Escat. Both approaches are planned for the NASA scatterometer (NSCAT) to be launched in early 1996 aboard the Japanese Advanced Earth Observing System (ADEOS) [3], [7].

Kennett and Li [3] examined SASS data to identify potential calibration sites, concluding that tropical rain forests such as the Amazon and Congo forests are remarkably homogeneous over a large area. However, these rain forest regions do exhibit some spatial and temporal variability [1], [3], [6]. This variability, if unaccounted for, can lead to biases and increased uncertainty in the calibration of the scatterometer. In this paper we present a simple methodology for using extended-area targets for the calibration of spaceborne scatterometer data. The method is not designed for absolute calibration but is a relative calibration method designed to ensure consistency between measurements of $\sigma^{\circ}$ from all antennas over the entire swath. This recalibration was developed and applied as a result of observing inconsistencies in SASS measurements. It has also been applied to Escat data. Time-varying, beamdependent and cell-dependent calibration corrections of up to several tenths of a decibel for SASS result. After applying the corrections, the SASS measurements exhibit much improved consistency and less variability. We have found the Escat data to be much more stable than the SASS data with only very small corrections indicated.

In this paper we outline our methodology and present sample results for SASS and Escat. We first provide some relevant background information and then describe the instrument calibration model assumed in our technique. We briefly describe our method, identifying its limitations, and then provide sample results for SASS and Escat. Finally, we provide a summary conclusion.

\section{BACKGROUND}

Spaceborne wind scatterometers use multiple antennas to make $\sigma^{\circ}$ measurements at different azimuth angles. Each antenna produces an instantaneous footprint several hundred kilometers long and a few kilometers wide at a given azimuth angle relative to the satellite subtrack. These instantaneous footprints are resolved into smaller observation cells by means 
of Doppler filtering (SASS) or range gating (Escat) [7]. Thus, a given point of the observation swath is observed several times as the spacecraft moves along the ground track. However, the measurements may be at different incidence angles. This greatly complicates antenna beam-to-beam calibration using extended area targets since the incidence angle dependence of $\sigma^{\circ}$ must be accounted for. We note that while SASS made near-nadir $\sigma^{\circ}$ measurements, these are not considered in this paper.

To make a backscatter measurement the scatterometer transmits a pulse and measures the returned power $\left(\widehat{P_{R}}\right.$, the backscattered signal with additive noise) reflected from the surface. A separate noise-only power measurement $\widehat{P_{N}}$ is also made. To compute the backscattered signal power measurement $\widehat{P_{S}}$, the noise measurement is subtracted from the signal and noise measurement, i.e., $\widehat{P_{S}}=\widehat{P_{R}}-\widehat{P_{N}}$. The measured $\sigma^{\circ}, \widehat{\sigma^{\circ}}$, is computed (retrieved) from the signal power measurement using the radar equation

$$
\widehat{\sigma^{\circ}}=X \widehat{P_{S}}
$$

where

$$
X=\frac{(4 \pi)^{3} R^{4} G_{p}}{P_{T} \lambda^{2} L_{s} G^{2} A_{c}}
$$

where $R$ is the range from the scatterometer to the observation cell, $G_{p}$ is the processor gain, $P_{T}$ is the transmitter power, $\lambda$ is the radar wavelength, $L_{s}$ is the system loss, $G$ is the antenna gain, and $A_{c}$ is the resolution element (cell) area. In general, $\sigma^{\circ}$ is a function of the incidence angle $(\theta)$ of the measurements, with larger incidence angles generally producing smaller $\sigma^{\circ}$ values.

Any errors in the conversion of the raw (data number) measurements to power measurements or in determining the parameters of the radar equation will produce errors in $\widehat{\sigma^{\circ}}$. In addition, because the signal-and-noise and noise-only measurements are noisy, the signal power measurement (and the measured $\sigma^{\circ}$ ) is also noisy. The total variability of the $\sigma^{\circ}$ measurements may be described by $K_{P}$ which is defined as the normalized standard deviation of the measurements, i.e.,

$$
\begin{aligned}
K_{P} & =\frac{\left\{\operatorname{Var}\left[\widehat{P_{R}}\right]\right\}^{1 / 2}}{P_{R}} \\
& =\frac{\left\{\operatorname{Var}\left[\widehat{\sigma^{\circ}}\right]\right\}^{1 / 2}}{\sigma^{\circ}} .
\end{aligned}
$$

It is useful to decompose the total variability into three independent noise/variability contributions: communication error (radiometric noise), retrieval error, and modeling error, i.e.,

$$
K_{P}^{2}=K_{P c}^{2}+K_{P r}^{2}+K_{P m}^{2}
$$

where $K_{P c}$ is the contribution due to the communication error, $K_{P r}$ is the contribution due to the retrieval error, and $K_{P m}$ is the contribution due to the modeling error.

$K_{P_{c}}$ is a function of the measurement signal-to-noise ratio (SNR) which is a function of the instrument design, the geometry, and the $\sigma^{\circ}$ value [7]. For SASS, $K_{P_{c}}$ varies from $2 \%$ to as high as $400 \%$. For Escat, $K_{P c}$ has a nominal value of $5 \%$. This variability is inherent in the scatterometer measurements and is unaffected by calibration.

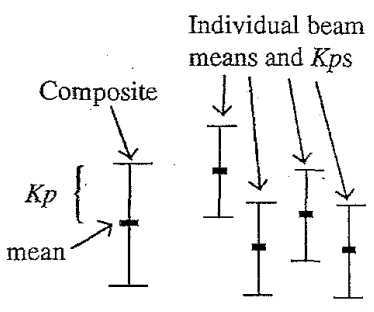

(a)

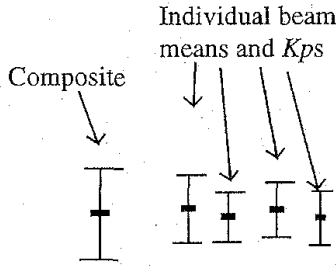

(b)
Fig. 1. Illustration of the individual and composite data means and standard deviations for each antenna (a) prior to and (b) after the calibration correction. The vertical axis is the $\sigma^{\circ}$ error. Horizontal placement is shown for clarity.

$K_{P r}$ is the normalized standard deviation of $\sigma^{\circ}$ due to errors in the values of the radar equation parameter $X$ and in the error in the conversion from raw data number to return power. These errors may be time-varying. One goal of calibration is to minimize these errors and thereby reduce the effective $K_{P r}$. There may also be fixed biases in the estimated $\sigma^{\circ}$ measurements due to biases in the values of the parameters in $X$. Another goal of calibration is to ensure that the measurements are consistent from antenna beam to beam over the swath, i.e., to minimize the measurement bias.

$K_{P m}$ describes the $\sigma^{\circ}$ variability inherent to the target. For example, over the ocean $K_{P m}$ is due to geophysical modeling. error, the variability of $\sigma^{\circ}$ for a given wind speed and direction. The rain forest also exhibits inherent $\sigma^{\circ}$ variability due to small variations in canopy density, leaf type and distribution, topography, and diumal and seasonal effects. By accounting for part of the spatial and temporal variability of $\sigma^{\circ}$ the total $K_{P m}$ may be reduced. The residual variability due to unmodeled effects is the limiting factor in the accuracy of spaceborne scatterometer calibration using the rain forest. As described later, we estimate that over the Amazon data mask described below, $K_{P m}$ is approximately $0.15 \mathrm{~dB}$.

To graphically illustrate the goal of our calibration technique, consider Fig. 1. Fig. 1(a) illustrates the observed means and standard deviations ( $K_{P}$ 's) of the $\sigma^{\circ}$ measurements (in decibels) at a given incidence angle from each of the antenna beams prior to postlaunch calibration. Fig. 1(b) illustrates the mean and standard deviations $\left(K_{P}\right.$ 's) with the calibration corrections derived below applied to the measurements. Each individual case corresponds to a given antenna beam and/or cell and/or time, etc. The composite mean and $K_{P}$ results when all the measurements are combined. By applying the calibration corrections the individual means are made consistent, eliminating beam-to-beam gain errors, and the composite $K_{P}$ is reduced. Note that $K_{P c}$ is unaffected by the calibration correction.

\section{METHOD DESCRIPTION}

Our ultimate interest in calibrating the scatterometer data is to obtain highly accurate measurements of $\sigma^{\circ}$. Such measurements must be consistent between the antennas and over the full measurement incidence (and azimuth) angle range. Inconsistencies arise due to calibration errors and uncorrected time-varying changes in the parameters making up $X$. Such errors and changes will result in errors in the inferred (mea- 


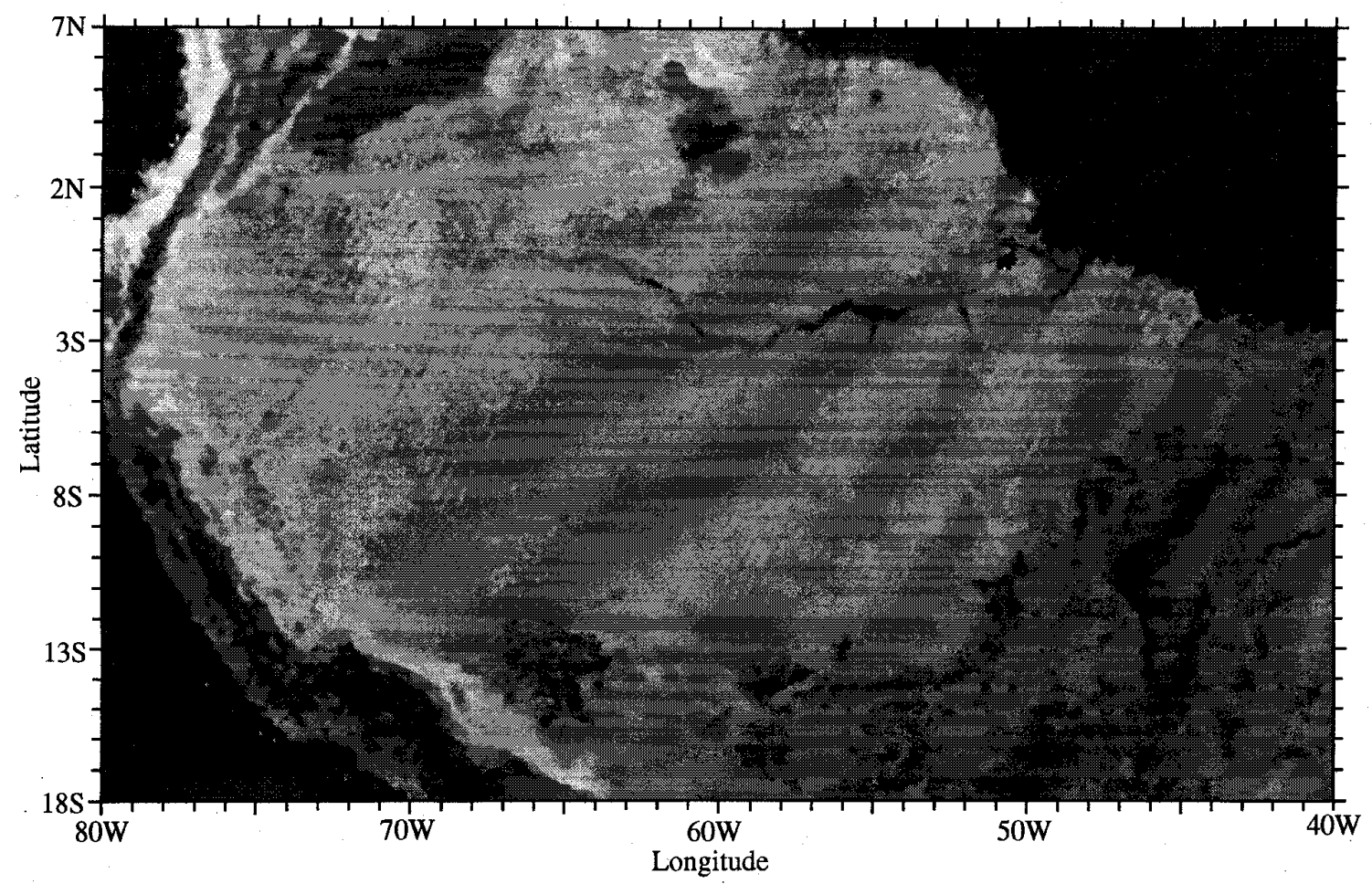

Fig. 2. Enhanced resolution $\mathcal{A}\left(\sigma^{\circ}\right.$ at a $40^{\circ}$ incidence angle) image of the Amazon Basin generated from 3 months of SASS data using the SIRF algorithm.

sured) value of $\sigma^{\circ}$. To model the error, a total error approach is adopted. The measured parameter is assumed to be $\sigma^{\circ}$ with all error sources (e.g., the error in the calculation of the received power from the raw data number and the error in the value of $X$ used to compute $\sigma^{\circ}$ from the power measurement) assumed to be multiplicative (which is equivalent to assuming errors are additive in log space). While this model ignores additive bias errors, we note that additive errors will generally affect $P_{R}$ and $P_{N}$ similarly and will be canceled when computing $P_{S}$.

In the following sections we describe our methodology for calibrating the scatterometer data. We first describe our approach for calibration modeling and estimating the gain calibration correction. We then describe our method of generating a spatial data selection mask and consider the issue of temporal variability. Finally, we describe the model for the dependence of $\sigma^{\circ}$ on incidence angle and the calculation of the gain correction. In later sections we describe the results of applying our method to SASS and Escat data.

\section{A. Measurement Model and Gain Correction Estimation}

Assuming that over the target area $\sigma^{\circ}$ is not a function of azimuth angle, the $n$th measurement of $\sigma^{\circ}$, denoted by $z_{n}$, is modeled (in decibels) as

$$
z_{n}=\sigma^{\circ}\left(L_{n}, t_{n}, \theta_{n}\right)+G_{e}\left(b_{n}, c_{n}, t_{n}, \theta_{n}\right)+\text { noise }_{n}
$$

where $\sigma^{\circ}\left(L_{n}, t_{n}, \theta_{n}\right)$ is the true value of $\sigma^{\circ}$ of the surface at location $L_{n}$ and time $t_{n}$ with observation incidence angle $\theta_{n}$ and $G_{e}\left(b_{n}, c_{n}, t_{n}, \theta_{n}\right)$ is the total end-to-end system gain error for antenna beam $b_{n}$ and cross-track cell number $c_{n}$ observed at incidence angle $\theta_{n} . G_{e}\left(b_{n}, c_{n}, t_{n}, \theta_{n}\right)$ is thus the net error in determining $\sigma^{\circ}$ from the raw power measurement. The zero mean noise term is due to unmodeled target variability and the inherent variability in the signal power measurement due to communication noise. Note that $c_{n}$ is included as an argument to $G_{e}$ to account for the differences in the channel gains of the scatterometer processor. Unlike Escat, each SASS resolution cell is processed as a separate channel. In both cases a cell spans a relatively small range of $\theta$.

For a perfectly calibrated, stable instrument, $G_{e}=0$. However, due to thermal effects, calibration errors, etc., $G_{e}$ varies with time, antenna, and channel number. Our goal is to determine an estimate, $\widehat{G_{e}}\left(b_{n}, c_{n}, t_{n}, \theta_{n}\right)$, to be applied (in decibels) to the measurements such that the expected value of the corrected $\sigma^{\circ}$ measurements, $\widehat{\sigma^{\circ}}\left(L_{n}, t_{n}, \theta_{n}\right)$

$$
\widehat{\sigma^{\circ}}\left(L_{n}, t_{n}, \theta_{n}\right)=z_{n}-\widehat{G_{e}}\left(b_{n}, c_{n}, t_{n}, \theta_{n}\right)
$$

and the actual value of $\sigma^{\circ}\left[\sigma^{\circ}\left(L_{n}, t_{n}, \theta_{n}\right)\right]$ correspond in the mean square sense, i.e., $\sigma^{\circ}\left(L_{n}, t_{n}, \theta_{n}\right)=\widehat{\sigma^{\circ}}\left(L_{n}, t_{n}, \theta_{n}\right)$ when there is no noise.

$\widehat{G_{e}}\left(b_{n}, c_{n}, t_{n}, \theta_{n}\right)$ can be decomposed into the sum of a fixed, absolute gain bias error $G_{\text {abs }}$ and a zero-average gain error term $G_{e}^{\prime}\left(b_{n}, c_{n}, t_{n}, \theta_{n}\right)$, i.e.,

$$
\widehat{G_{e}}\left(b_{n}, c_{n}, t_{n}, \theta_{n}\right)=G_{\mathrm{abs}}(\theta)+G_{e}^{\prime}\left(b_{n}, c_{n}, t_{n}, \theta_{n}\right)
$$

where the average is over all beams, cells, time, and incidence angles. Unfortunately, because of the lack of ground truth there is no accurate way to determine $G_{\mathrm{abs}}(\theta)$ since this requires knowledge of the absolute $\sigma^{\circ}$ of the rain forest as a function of incidence angle. Absolute calibration requires either ground 


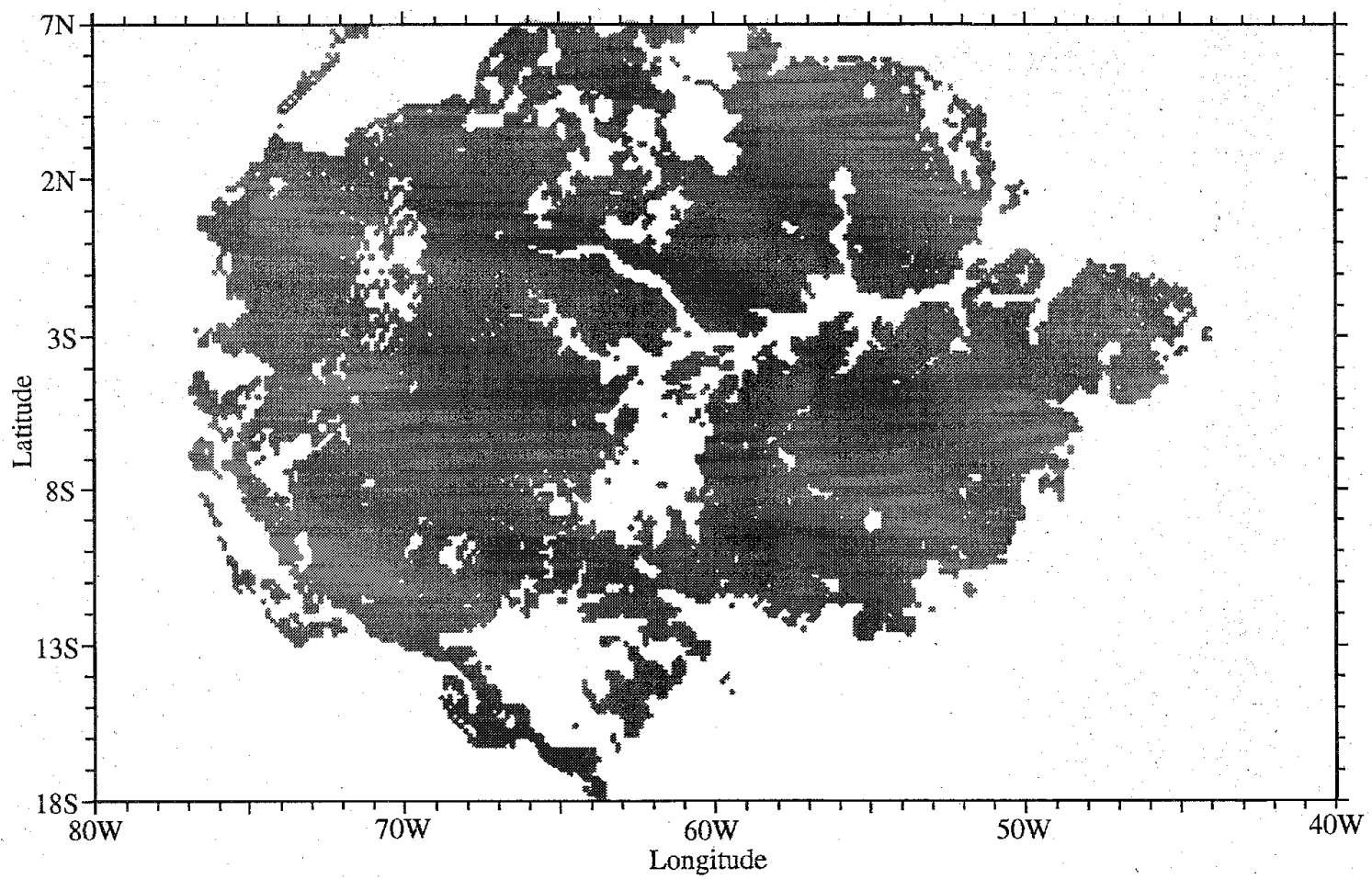

Fig. 3. Data mask used for selection of SASS data from over the Amazon rain forest. Only measurements which fall completely within the mask are used in this study. This mask corresponds to pixels with $\mathcal{A}=8.0 \pm 0.5 \mathrm{~dB}$ from Fig. 2.

stations to directly measure the instrument and/or aircraft overflights to measure the $\sigma^{\circ}$ of the rain forest. In this paper we concentrate on determining the relative gain error term $G_{e}^{\prime}\left(b_{n}, c_{n}, t_{n}, \theta_{n}\right)$, the average value of which is assumed to be zero. The resulting gain correction will be accurate to within $G_{\text {abs }}(\theta)$. Ignoring $G_{\text {abs }}(\theta)$, (1) becomes

$$
\widehat{\sigma^{\circ}}\left(L_{n}, t_{n}, \theta_{n}\right)=z_{n}-G_{e}^{\prime}\left(b_{n}, c_{n}, t_{n}, \theta_{n}\right) \text {. }
$$

In principle, to determine $G_{e}^{\prime}\left(b_{n}, c_{n}, t_{n}, \theta_{n}\right)$ we must know the true $\sigma^{\circ}\left(L_{n}, t_{n}, \theta_{n}\right)$. However, this is impractical due to the difficulty of obtaining sufficient ground truth measurements. Instead, we estimate $\sigma^{\circ}\left(L_{n}, t_{n}, \theta_{n}\right)$ from the noisy measurements $z_{n}$. To do this we assume that $G_{e}^{\prime}\left(b_{n}, c_{n}, t_{n}, \theta_{n}\right)$ is independent of $\sigma^{\circ}\left(L_{n}, t_{n}, \theta_{n}\right)$. The calibration problem may be stated as follows: Given measurements $z_{n}$ and the measurement model

$$
z_{n}=\sigma^{\circ}\left(L_{n}, t_{n}, \theta_{n}\right)+G_{e}^{\prime}\left(b_{n}, c_{n}, t_{n}, \theta_{n}\right)+\text { noise }_{n}
$$

estimate $G_{e}^{\prime}\left(b_{n}, c_{n}, t_{n}, \theta_{n}\right)$ such that $\widehat{\sigma^{\circ}}\left(L_{n}, t_{n}, \theta_{n}\right)-$ $\sigma^{\circ}\left(L_{n}, t_{n}, \theta_{n}\right)$ is minimized in the mean-squared sense.

To simplify the solution of this problem the location dependence of $\sigma^{\circ}$ is eliminated by choosing a large target region which exhibits a homogeneous response in which $\sigma^{\circ}$ is not dependent on azimuth angle. A large region is required to ensure that an adequate number of measurements are available to estimate $G_{e}^{\prime}\left(b_{n}, c_{n}, t_{n}, \theta_{n}\right)$. As described below, the Amazon rain forest is a suitable calibration target.

Unfortunately, both the $\sigma^{\circ}$ of the rain forest and the system gain error $G_{e}^{\prime}$ may vary with time. While some diurnal

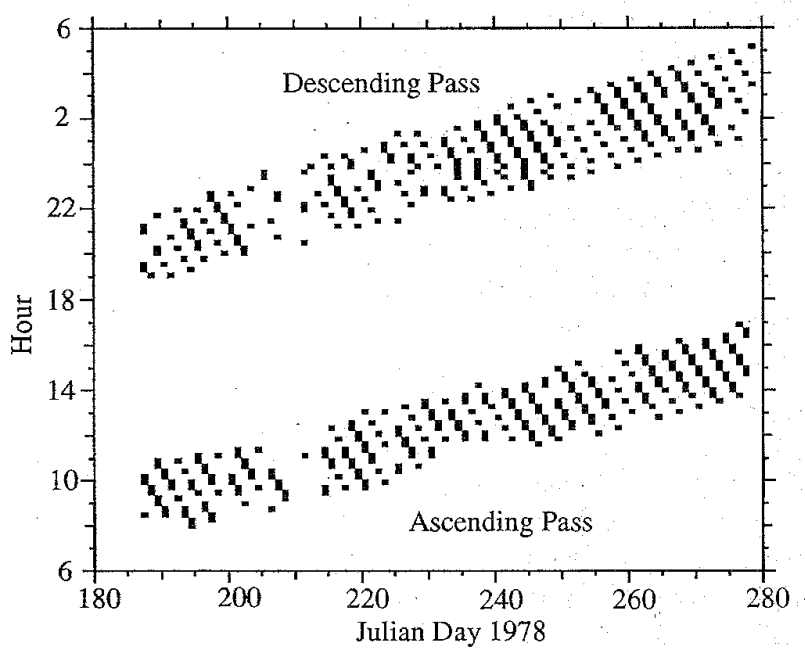

Fig. 4. Measurement time-of-day versus day for SASS measurements over the data mask.

variability in $\sigma^{\circ}$ may occur (which results in slightly different observed values of $\sigma^{\circ}$ for ascending and descending passes as discussed later), the $\sigma^{\circ}$ of the rain forest is expected to be only seasonally dependent and thus change only very slowly with time. The instrument system gain error $G_{e}^{\prime}$ also exhibits variability on both short and long time scales; however, in this research we concentrate on scales of variability greater than several days. Variability in $\sigma^{\circ}$ and $G_{e}^{\prime}$ on time scales shorter than this are treated as noise. As discussed later, using only scatterometer measurements does not allow for a unique 


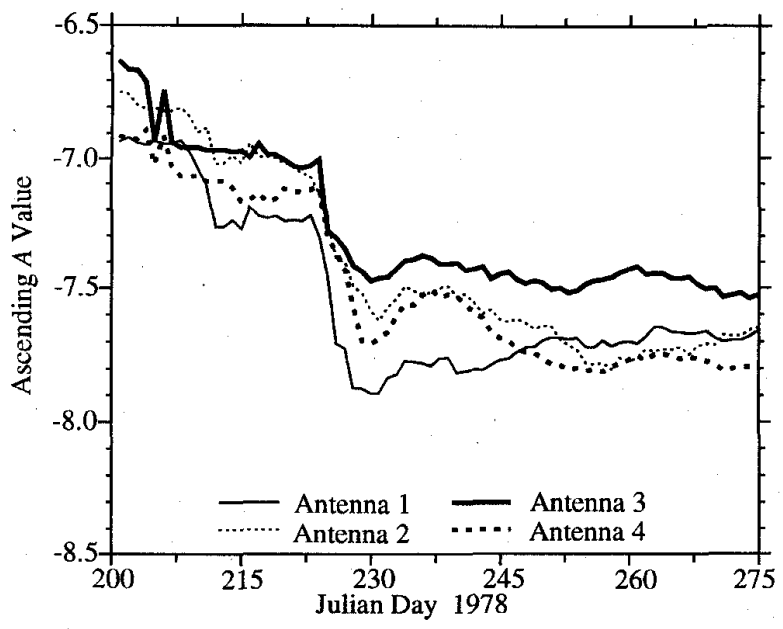

(a)

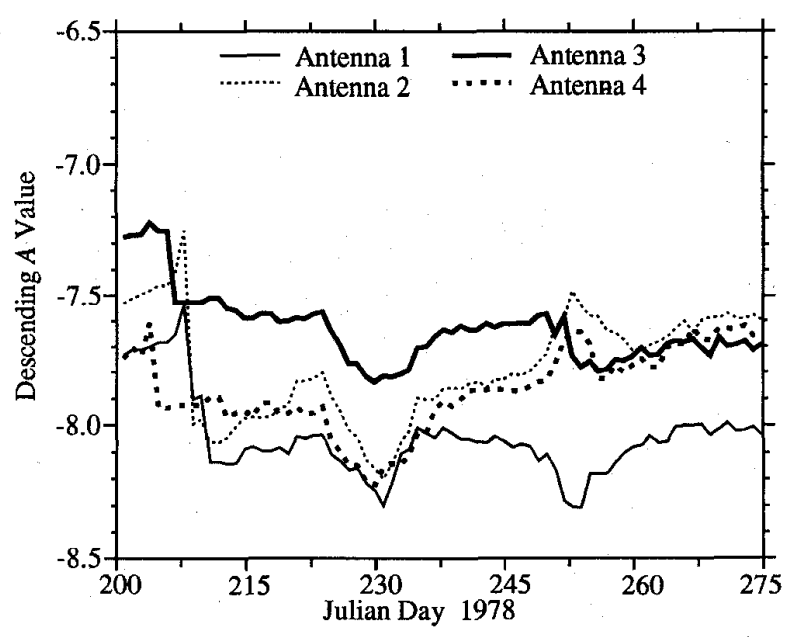

(b)

Fig. 5. Plots of SASS $\mathcal{A}$ coefficients as a function of time for the (a) ascending and (b) descending passes. To compute each coefficient, an eight-day sliding window, with the window centered at the plotted point, is used.

separation of the long-term variability of the rain forest and instrument.

Over our calibration target, and assuming time stationarity for the moment, (3) can be written as

$$
\begin{aligned}
z_{n} & =\sigma^{\circ}\left(\theta_{n}\right)+G_{e}^{\prime}\left(b_{n}, c_{n}, \theta_{n}\right)+\text { noise }_{n} \\
& =F\left(b_{n}, c_{n}, \theta_{n}\right)+\text { noise }_{n}
\end{aligned}
$$

where $\sigma^{\circ}\left(\theta_{n}\right)$ describes the dependency of $\sigma^{\circ}$ on the incidence angle for the target and $F\left(b_{n}, c_{n}, \theta_{n}\right)=\sigma^{\circ}\left(\theta_{n}\right)+$ $G_{e}^{\prime}\left(b_{n}, c_{n}, \theta_{n}\right)$ is a function (to be estimated) of the beam number, cell number, and incidence angle.

To determine $G_{e}^{\prime}\left(b_{n}, c_{n}, \theta_{n}\right)$, we first determine $\bar{F}\left(b_{n}, \theta_{n}\right)$ for each antenna $\left(b_{n}\right)$ where $\bar{F}\left(b_{n}, \theta_{n}\right)=\left\langle F\left(b_{n}, c_{n}, \theta_{n}\right)\right\rangle$ is the average over $c_{n}$ of $F\left(b_{n}, c_{n}, \theta_{n}\right)$. Let $F^{\prime}(\theta)=$ $\left\langle\bar{F}\left(b_{n}, \theta_{n}\right)\right\rangle$ be the average of $\bar{F}\left(b_{n}, \theta_{n}\right)$ over all $b_{n}$. Since $G_{e}^{\prime}\left(b_{n}, c_{n}, \theta_{n}\right)$ averaged over all beams and cells is assumed to be zero, it follows that $\sigma^{\circ}(\theta)=F^{\prime}(\theta)$. Determination of $F^{\prime}(\theta)$ is described later.

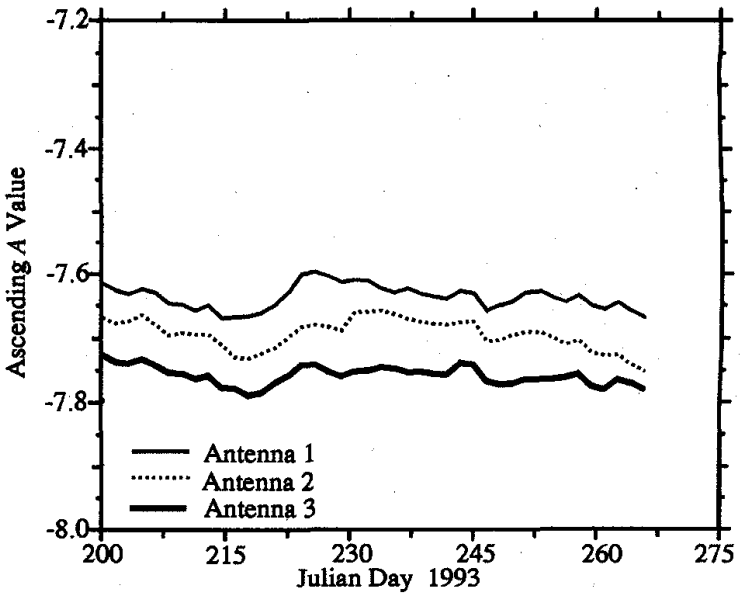

(a)

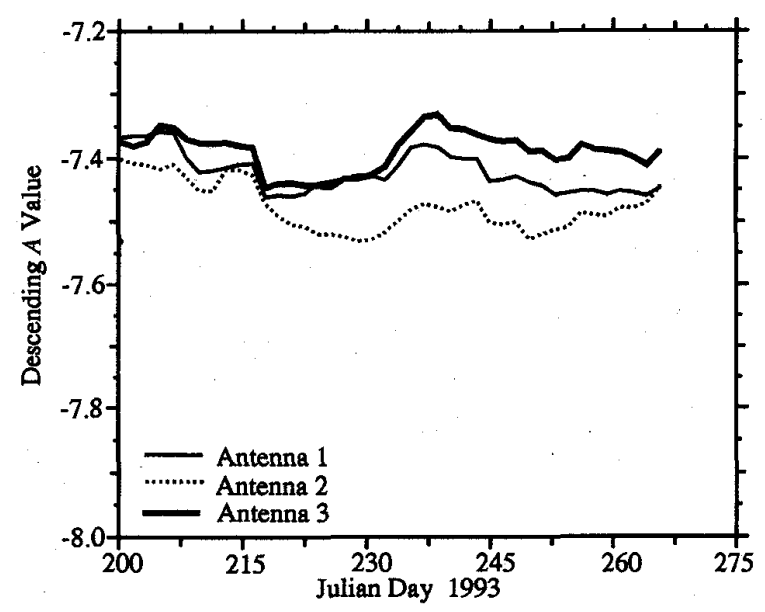

(b)

Fig. 6. Plots of Escat $\mathcal{A}$ coefficients as a function of time for the (a) ascending and (b) descending passes. To compute each coefficient, a sixteen-day sliding window, with the window centered at the plotted point, is used.

Once $\sigma^{\circ}(\theta)$ is determined, estimating $G_{e}^{\prime}\left(b_{n}, c_{n}, \theta_{n}\right)$ is straight-forward using

$$
\begin{aligned}
G_{e}^{\prime}\left(b_{n}, c_{n}, \theta_{n}\right) & =\left\langle z_{n}-\sigma^{\circ}(\theta)\right\rangle \\
& =\left\langle z_{n}-F^{\prime}(\theta)\right\rangle .
\end{aligned}
$$

To aid in computing $F^{\prime}(\theta)$, we assume a parametric model relating $\sigma^{\circ}$ and $\theta$ and estimate the parameters of the model from the measurements $z_{n}$. In the following sections we describe our methodology for selecting a homogeneous target area, dealing with time variations in the target response, computing $\bar{F}\left(b_{n}, \theta_{n}\right)$ and $F^{\prime}(\theta)$, and determining $G_{e}^{\prime}\left(b_{n}, c_{n}, \theta_{n}\right)$.

\section{B. Spatial Data Selection Mask}

As demonstrated in various studies [1], [3], [6], the Amazon tropical rain forest is remarkably homogeneous and exhibits limited seasonal variation. However, the forest does exhibit some spatial and temporal variability [6]. Such variability can adversely impact the scatterometer calibration. Previous researchers [1] have used a data selection mask based on 
vegetation maps in order to select a region of spatially homogeneous response. The coarse resolution and limitations of the vegetation maps limit the accuracy of this approach. In this research a data mask is generated directly from the $\sigma^{\circ}$ measurements. The mask is generated with the aid of a resolution enhancement algorithm known as SIRF (Scatterometer Image Reconsutrction with Filtering) [5], [6]. By using SIRF to generate an enhanced resolution map of the radar backscatter, a more precise selection of the area of homogeneous radar response is possible.

The SIRF algorithm generates images of the incidence angle-normalized $\sigma^{\circ}(\mathcal{A})$ and the incidence angle slope $(\mathcal{B})$ from the $\sigma^{\circ}$ measurements where

$$
10 \log _{10} \sigma^{\circ}=\mathcal{A}+\mathcal{B} \vartheta
$$

where $\vartheta=\theta-40^{\circ}$. Note that $40^{\circ}$ is the mean incidence angle of the measurements. In effect, $A$ is the value of $\sigma^{\circ}$ at $40^{\circ}$ incidence angle. This simple model for the response of $\sigma^{\circ}$ as a function of incidence angle has been used extensively by previous investigators (e.g., [1], [3], [6]). In this study this model is only used for the generation of the data mask. A higher order polynomial is used for the calibration procedure.

SIRF generates images with 3 - to $4-\mathrm{km}$ resolution (SASS, $25-\mathrm{km}$ resolution for Escat) from the nominally 50 - $\mathrm{km}$ resolution $\sigma^{\circ}$ measurements. The SIRF algorithm, originally developed for SASS but applicable to Escat, is described in detail in [5] and has been successfully used in studies of tropical vegetation [6] and polar ice [4], [9]. To illustrate, Fig. 2 shows an $\mathcal{A}$ image generated from 3 months of SASS data. Note the remarkably homogeneous response of the rain forest region.

To generate the selection mask from the $\mathcal{A}$ image, a contiguous area with an $\mathcal{A}$ response within $\pm 0.5 \mathrm{~dB}$ of the mean Amazon tropical rain forest response $(\mathcal{A}=8.0 \mathrm{~dB}$ for $\mathrm{Ku}$ band and $\mathcal{A}=7.6 \mathrm{~dB}$ for $\mathrm{C}$-band) is selected. Only $\sigma^{\circ}$ measurements whose spatial extent fall completely within this region are used in the calibration procedure. For convenience, the final data mask resolution is reduced to $1 / 6^{\circ} \times 1 / 6^{\circ}$. The data mask approximately corresponds to the boundaries of the Amazon tropical rain forest but excludes woodland areas, floodplains, rivers, and small regions of degraded forest and savanna within the main rain forest area [6]. To illustrate, Fig. 3 shows the SASS data selection mask generated from Fig. 2. For the three summer months used, over 100000 measurements fall within the mask.

In generating this SIRF image, three months of data were used. The months (July-September) correspond to the full SASS mission in 1978. The corresponding months of 1993 were used for Escat to enable comparison of the algorithm with both sensors. The SIRF $\mathcal{A}$ images generated from the three months of data are, in effect, a temporal average over the period. After determining the calibration correction using the procedure described below, a second SIRF $\mathcal{A}$ image is generated using the corrected $\sigma^{\circ}$ values. A new data selection mask is then generated from the second $\mathcal{A}$ image. The resulting mask is virtually identical to the original mask with only small changes along the boundary of the region. The calibration corrections resulting from using the updated mask are virtually identical to those computed from the original mask. Masks generated from SASS and Escat data were similar.

\section{Temporal Variability}

The data selection mask results in a spatially homogeneous data set. However, temporal variability in the measurements is evident for both SASS and Escat. On short time scales, the ascending (north-bound) and descending (south-bound) passes occur at different times of day (the SASS measurement time of day in GMT is shown in Fig. 4). This limits our ability to study diumal variations. Because a small difference (for reasons not well understood - see later discussion in Section IV) in the response for ascending and descending passes is noted for both SASS and Escat, the ascending and descending passes are treated separately in the computation of $G_{e}^{\prime}\left(b_{n}, c_{n}, \theta_{n}\right)$.

Long term temporal variations in the $\mathcal{A}$ response (several tenths of a decibel) are evident in the data from both SASS and Escat (see Figs. 5 and 6). These can be attributed to either variations in the instrument gain or in the rain forest response. Without independent measurements of the $\sigma^{\circ}$ of the rain forest it is impossible to determine which changed. However, for SASS, we note significant (several tenths of a decibel) differences between the mean responses measured by the different antenna beams. Because little seasonal change in the rain forest is expected over the time period observed, we attribute the temporal variations primarily to long-term variations in the instrument gain and to correct them to a timefixed forest response. Nevertheless, the inability to uniquely distinguish between seasonal changes in the forest response and slow changes in the instrument calibration is a significant short-coming of our simple method. We note that the temporal variations observed for Escat are much smaller than for SASS (compare Figs. 5 and 6), suggesting that the Amazon response is, indeed, stable over the study period.

In order to deal with long-term temporal variations, the observation period was segmented into overlapping temporal windows. After considering various window lengths, an eightday window length was adopted for SASS [8]. To provide similar spatial coverage, a sixteen-day window length was adopted for Escat. In application, $\bar{F}\left(b_{n}, \theta_{n}\right)$ and the corresponding $G_{e}^{\prime}\left(b_{n}, c_{n}, \theta_{n}\right)$ are computed for each window position. The computed parameters are used to determine the corrected $\sigma^{\circ}$ values (2) for the one-day period in the center of the window. There are many thousands of $\sigma^{\circ}$ measurements within each temporal window position.

\section{Computation of $\bar{F}\left(b_{n}, \theta_{n}\right)$ and $G_{e}^{\prime}\left(b_{n}, c_{n}, \theta_{n}\right)$}

In order to determine $\bar{F}\left(b_{n}, \theta_{n}\right)$, the measurements falling within the selection mask are first used to estimate the parameters of a parametric model for the incidence angle response for each individual antenna. For simplicity, a fourth-order polynomial model in $\theta$ for $\sigma^{\circ}$ in $\mathrm{dB}$ has been adopted

$$
\bar{F}(b, \theta)=\mathcal{A}_{b}+\mathcal{B}_{b} \vartheta+\mathcal{C}_{b} \vartheta^{2}+\mathcal{D}_{b} \vartheta^{3}+\mathcal{E}_{b} \vartheta^{4}
$$

This simplistic model is consistent with the idea that $\sigma^{\circ}$ is a smooth function of incidence angle. Other polynomial orders were considered but a fourth order provides the maximum 


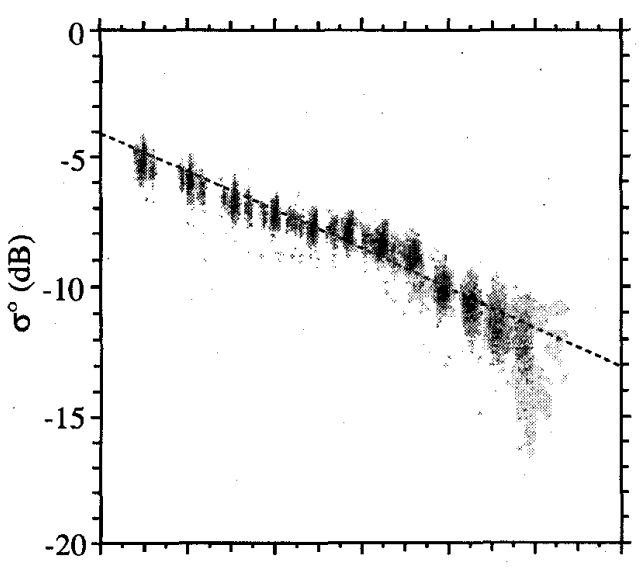

(a)

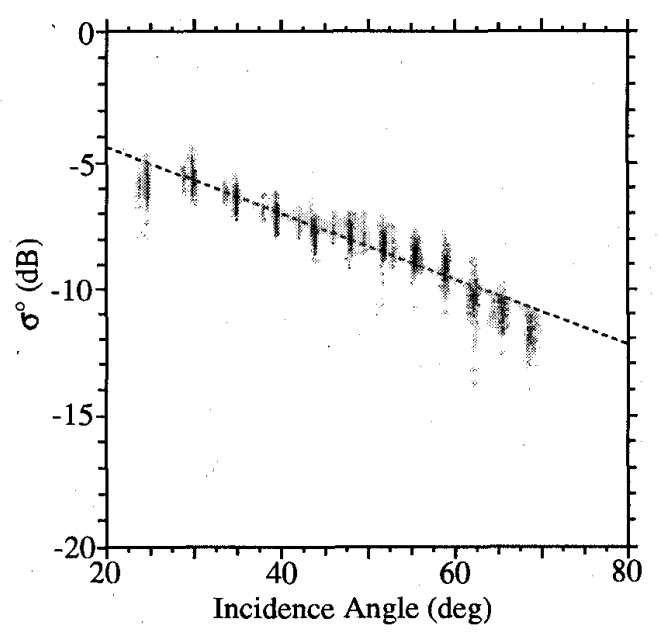

(c)

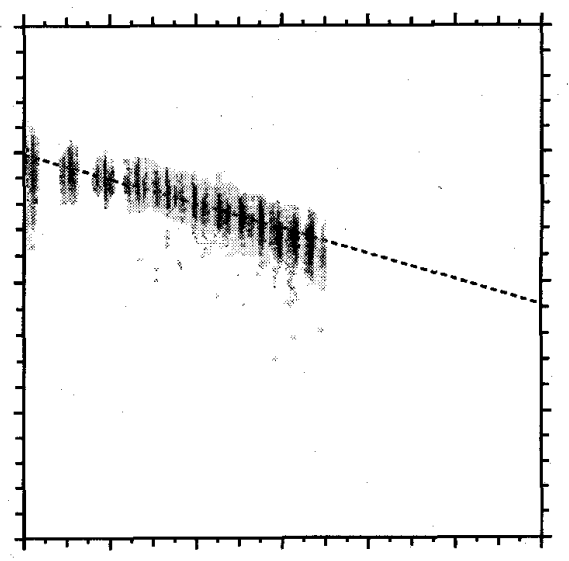

(b)

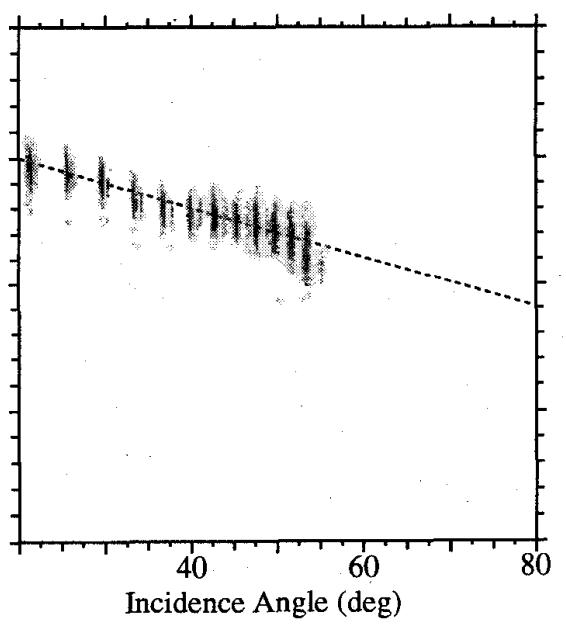

(d)

Fig. 7. Scatter plots of SASS $\sigma^{\circ}$ versus incidence angle for each antenna, ascending pass. (a) Antenna 1. (b) Antenna 2. (c) Antenna 3. (d) Antenna 4. Because of the number of measurements, the values of $\sigma^{\circ}$ and $\theta$ are quantized into bins. The gray values in each bin are indicative of the number of $\sigma^{\circ}$ measurements in each bin.

information with a minimum number of coefficients for SASS data [8]. The polynomial coefficients for each antenna are separately estimated from the measurements $z_{n}$ using weighted regression. The regression weights are the $K_{P c}$ 's of the $\sigma^{\circ}$ measurements. The mean $\sigma^{\circ}$ response $\left[F^{\prime}(\theta)=\sigma^{\circ}(\theta)\right]$ is then

$$
F^{\prime}(\theta)=\mathcal{A}+\mathcal{B} \vartheta+\mathcal{C} \vartheta^{2}+\mathcal{D} \vartheta^{3}+\mathcal{E} \vartheta^{4}
$$

where the coefficients are the average of the coefficients in (5), e.g.,

$$
\mathcal{A}=\frac{1}{N_{b}} \sum_{n=1}^{N_{b}} \mathcal{A}_{b} .
$$

$G_{e}^{\prime}\left(b_{n}, c_{n}, \theta_{n}\right)$ is then computed using (4) where $\sigma^{\circ}(\theta)=$ $F^{\prime}(\theta)$. If desired, $G_{e}^{\prime}\left(b_{n}, c_{n}, \theta_{n}\right)$ can be decomposed into a beam-to-beam relative gain error term $G_{b}\left(b_{n}\right)$, an antenna pattern correction term $G_{p}\left(b_{n}, \theta\right)$, and (for SASS) a cell gain term $G_{c}\left(c_{n}\right)$,

$$
G_{e}^{\prime}\left(b_{n}, c_{n}, \theta_{n}\right)=G_{b}\left(b_{n}\right)+G_{p}\left(b_{n}, \theta\right)+G_{c}\left(c_{n}\right) .
$$

To compute these terms $G_{b}\left(b_{n}\right)$ is first computed as $G_{b}\left(b_{n}\right)=\left\langle G_{e}^{\prime}\left(b_{n}, c_{n}, \theta_{n}\right)\right\rangle$ where the average is over $c_{n}$ and $\theta_{n}$. $G_{p}\left(b_{n}, \theta\right)$ is computed as $G_{p}\left(b_{n}, \theta\right)=\left\langle G_{e}^{\prime}\left(b_{n}, c_{n}, \theta_{n}\right)-G_{b}\left(b_{n}\right)\right\rangle$ where the average is over $c_{n}$. Finally, $G_{c}\left(b_{n}, \theta\right)$ is computed as $G_{c}\left(b_{n}, \theta\right)=\left\langle G_{e}^{\prime}\left(b_{n}, c_{n}, \theta_{n}\right)-G_{b}\left(b_{n}\right)-G_{p}\left(b_{n}, \theta\right)\right\rangle$ where the average is over $b_{n}$ and $\theta_{n} . G_{e}^{\prime}\left(b_{n}, c_{n}, \theta_{n}\right)$ and its decompositions are illustrated for SASS and Escat in the following sections.

\section{SASS RESULTS}

This study was originally undertaken as a result of observing temporally varying inconsistencies in SASS measurements between antenna beams. To illustrate the observed inconsistencies, Figs. 7 and 8 show scatter plots of $\sigma^{\circ}$ versus incidence angle for antennas 1 and 2 separated into ascending and descending passes, respectively, over the Amazon rain forest. All $\sigma^{\circ}$ measurements within the data selection mask over the 


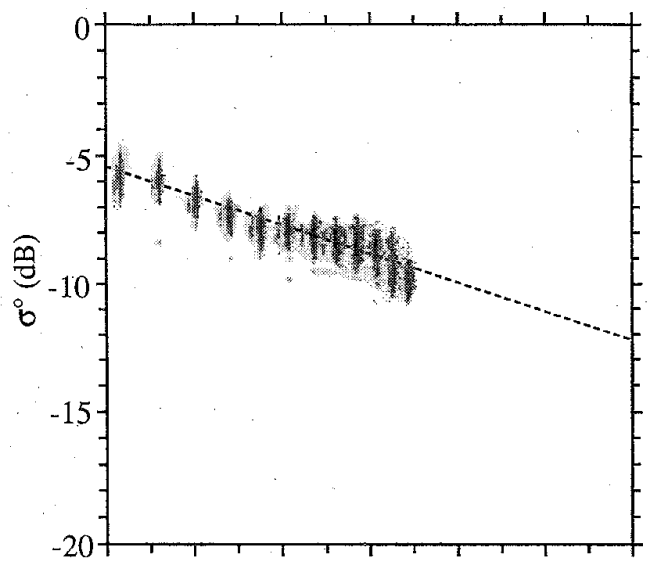

(a)

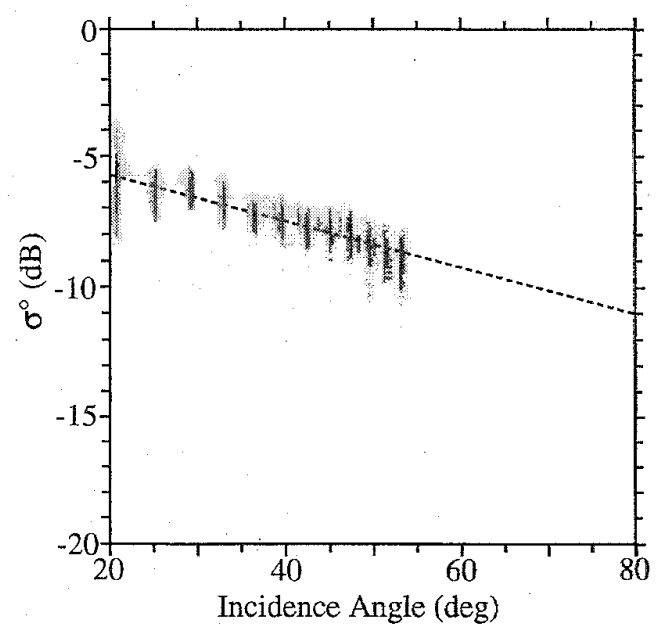

(c)

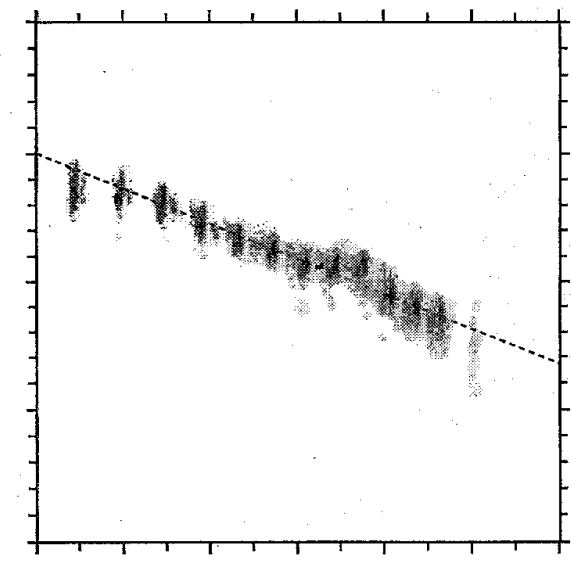

(b)

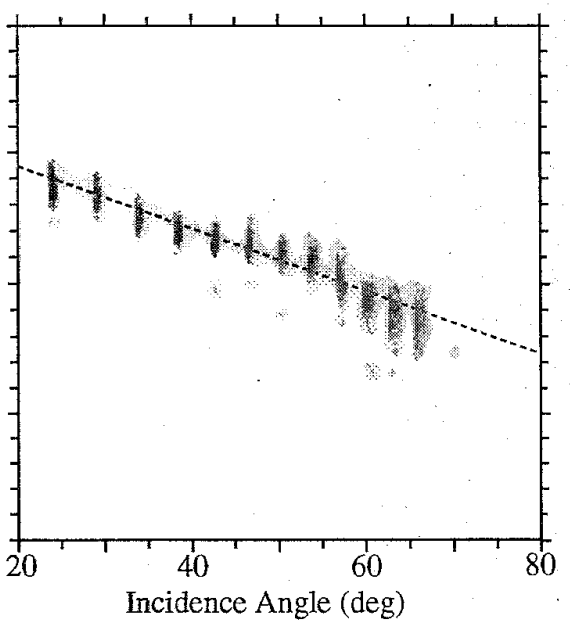

(d)

Fig. 8. Scatter plots of SASS $\sigma^{\circ}$ versus incidence angle for each antenna, descending pass. (a) Antenna 1. (b) Antenna 2. (c) Antenna 3. (d) Antenna 4. Because of the number of measurements, the values of $\sigma^{\circ}$ and $\theta$ are quantized into bins. The gray values in each bin are indicative of the number of $\sigma^{\circ}$ measurements in each bin.

three month mission are shown in these plots. For reference, a linear regression (in decibels) to the measurements is shown as a sloped line on the plots. Note the significant differences between antennas and ascending and descending passes.

To illustrate the observed temporal variations, the $\mathcal{A}$ coefficient of the $\sigma^{\circ}(\theta)$ response model described above (5) is plotted as a function of time in Fig. 5. We note differences in the mean $\mathcal{A}$ value between the beams as well as differences between the ascending and descending passes. Since the ascending pass occurs during the early morning hours this has been attributed to the accumulation of early morning dew on the forest leaves by Birrer et al. [1] based on the first few weeks of SASS data. Alternatively, the difference may be due to changes in the average orientation of leaves in the canopy at different sun angles or to differing instrument temperatures on the ascending and descending passes. We note that there is a significant step $(0.5 \mathrm{~dB})$ in the $\mathcal{A}$ value at Julian day (JD) 227 in the ascending data. A small step is also evident at JD 209 in both ascending and descending passes. No mechanism for a sudden change in the forest backscatter can be identified, suggesting that these steps are instrument gain related. To support this hypothesis, Fig. 9 illustrates the higher order $\mathcal{B}$ through $\mathcal{E}$ coefficients for the ascending pass. While small step changes in the higher order coefficients are evident at JD 209, only small bumps are evident at JD 227, suggesting that the change in $\mathcal{A}$ is not due to a change in the scattering mechanism but rather to a shift in the average system gain. Plots of the full model response $\bar{F}\left(b_{n}, \theta\right)$ for all antennas are shown in Fig. 10. The one $\sigma$ regression error for a given temporal window position is typically better than $0.09 \mathrm{~dB}$ with $r^{2}$ values of $60-95 \%$

Applying the method described above, calibration corrections $\left[G_{e}^{\prime}\left(b_{n}, c_{n}, \theta_{n}\right)\right]$ are determined to adjust the response from each beam to the average value between beams and over time. By way of illustration, a plot of $\left\langle G_{e}^{\prime}\left(b_{n}=1, c_{n}, \theta=\right.\right.$ $\left.\left.40^{\circ}\right)\right\rangle\left(G_{e}^{\prime}\right.$ averaged over $c_{n}$ for antenna 1 , corresponding to the correction in $\mathcal{A}$ ) as a function of time is shown in Fig. 11. The temporal variation in the calibration correction is typically less than $\pm 0.2 \mathrm{~dB}$ though the correction as a function of incidence angle may be as large as $0.4 \mathrm{~dB}$. The SASS cell 


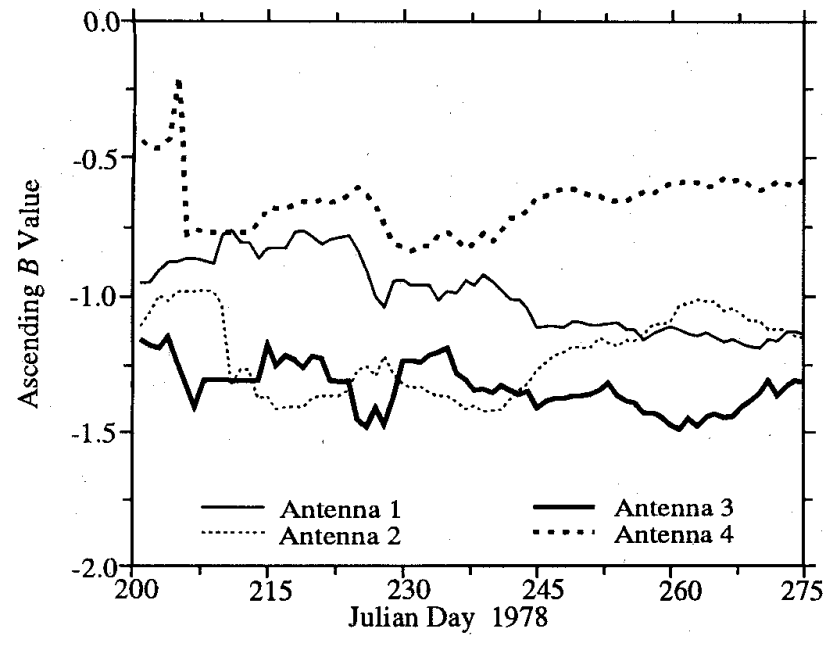

(a)

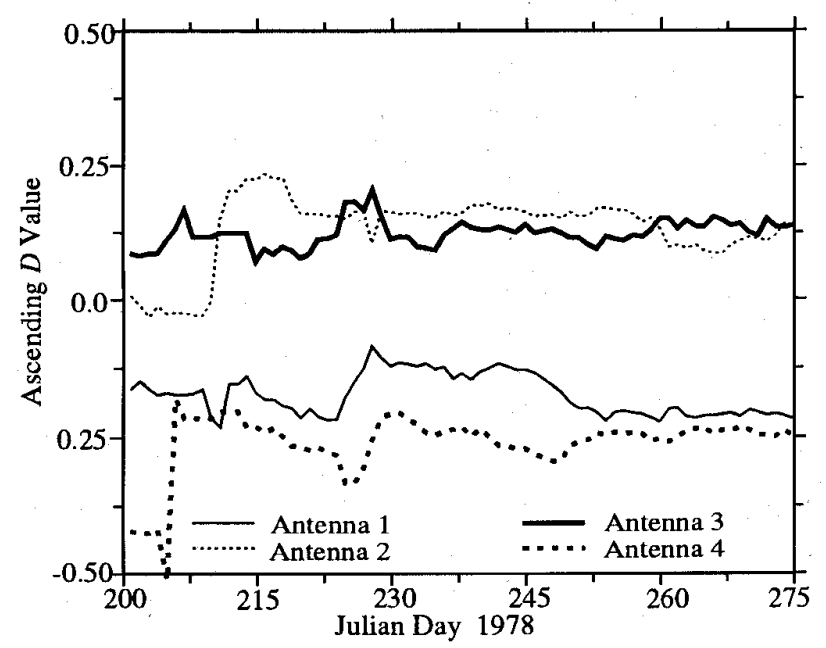

(c)

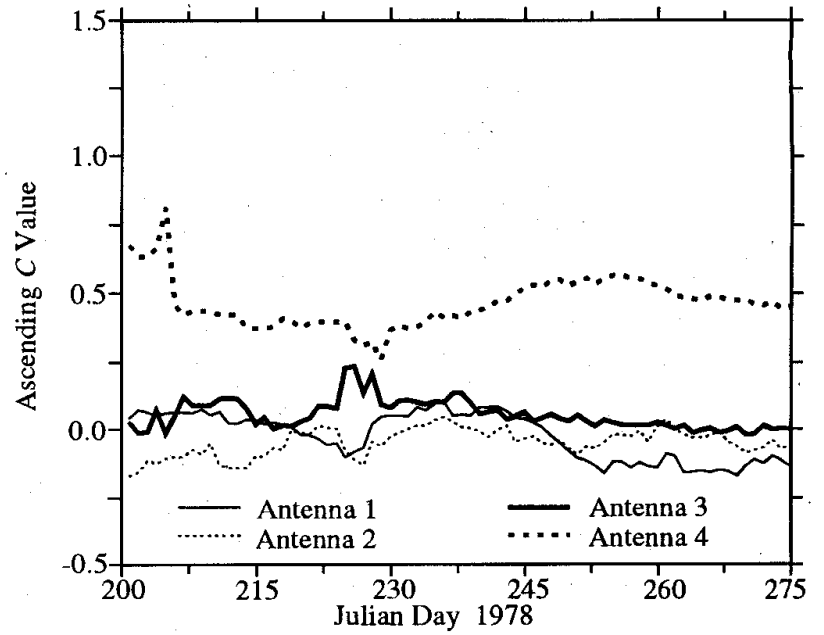

(b)

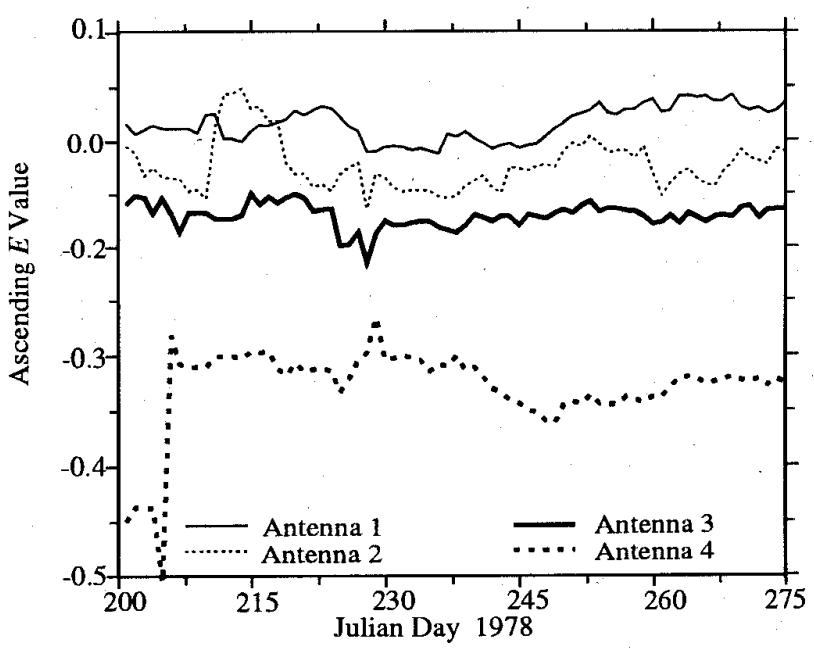

(d)

Fig. 9. Plots of SASS (a) $\mathcal{B}$, (b) $\mathcal{C}$, (c) $\mathcal{D}$, and (d) $\mathcal{E}$ coefficients as a function of time. To compute each coefficient, an eight-day sliding window, with the window centered at the plotted point, is used to compute the values shown.

gain calibration corrections $G_{c}\left(c_{n}\right)$ are illustrated in Fig. 12. The maximum absolute cell gain correction is less than 0.2 $\mathrm{dB}$ and varies with time by less than $\pm 0.05 \mathrm{~dB}$. While these corrections are relatively small, they may be important in some studies.

The one $\sigma$ regression error for the full three-month corrected data set provides an estimate of the average total $K_{P}$ of the SASS measurements. When the $\mathrm{rms} K_{P c}$ is removed, the residual $K_{P}$ is approximately $0.15 \mathrm{~dB}$ and corresponds to the Ku-band $K_{P m}$ of the Amazon forest. This value suggests that it is not possible to calibrate $\mathrm{Ku}$-band data to better than $\pm 0.15 \mathrm{~dB}$ using Amazon tropical rain forest data. This result is consistent with estimates given by Kennett and Li [3].

\section{ESCAT RESULTS}

Before considering Escat results, we need to make several observations. First, the primary scattering mechanisms of SASS and Escat $\sigma^{\circ}$ measurements are different. While $\mathrm{Ku}-$ band (SASS) signals have very little canopy penetration, C-band (Escat) exhibits significant canopy penetration. As a result Ku-band scattering is primarily surface scattering from the upper canopy while $\mathrm{C}$-band is primarily volume scattering, resulting in a different $\sigma^{\circ}$ versus incidence angle roll off. Second, the time of day of the two data sets are different and the data sets are separated by sixteen years. These differences complicate a direct comparison of the $\sigma^{\circ}$ values and the data mask. Thus, we concentrate on the consistency and stability of the measurements. Finally, the measurement cell response characteristics of Escat are quite different than SASS, limiting the degree of resolution enhancement possible for the Escat data (see [2], [5]). While SIRF is able to achieve 3- to 4-km resolution for SASS, the best possible resolution for Escat is estimated to be $25 \mathrm{~km}$ [2]. This limits the resolution of the data selection mask. Even so, the Escat data selection mask (not shown) is remarkably similar to the SASS mask shown in Fig. 3. 


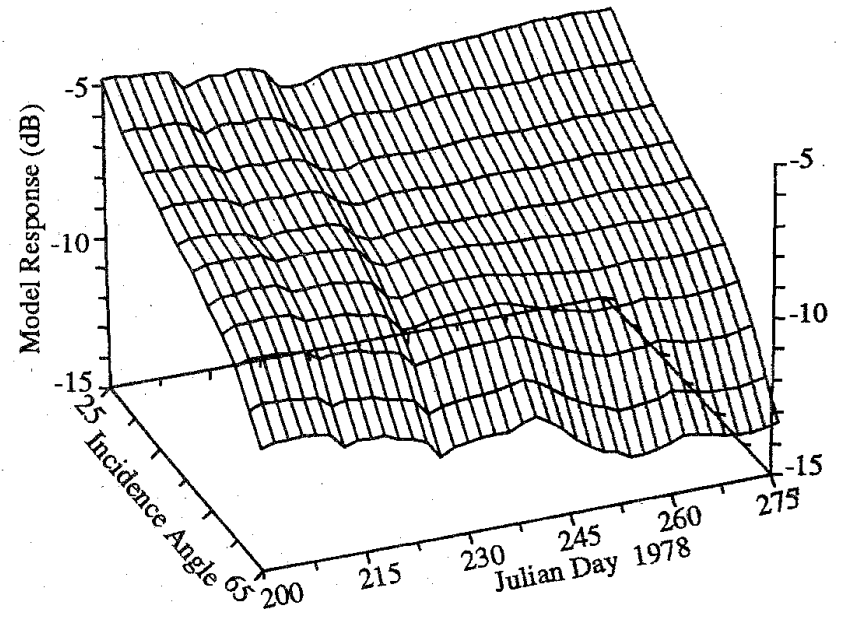

(a)

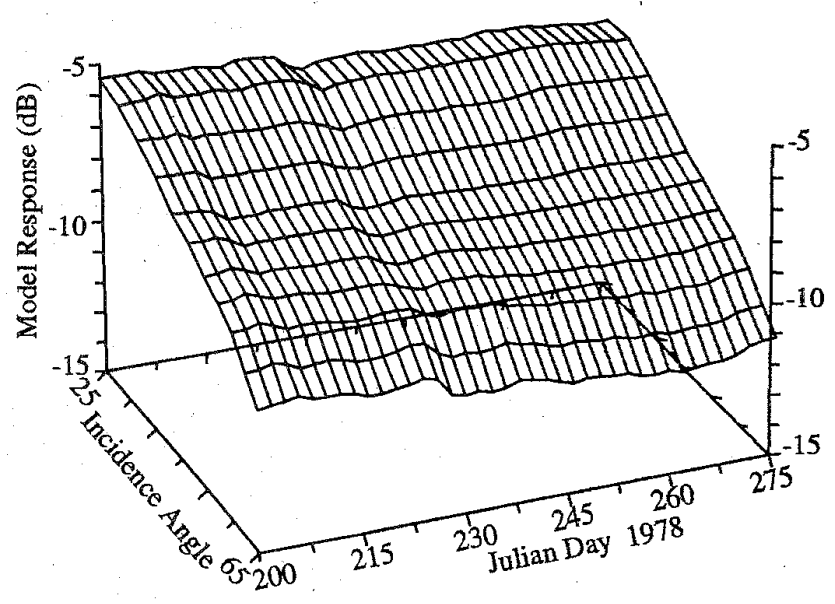

(c)

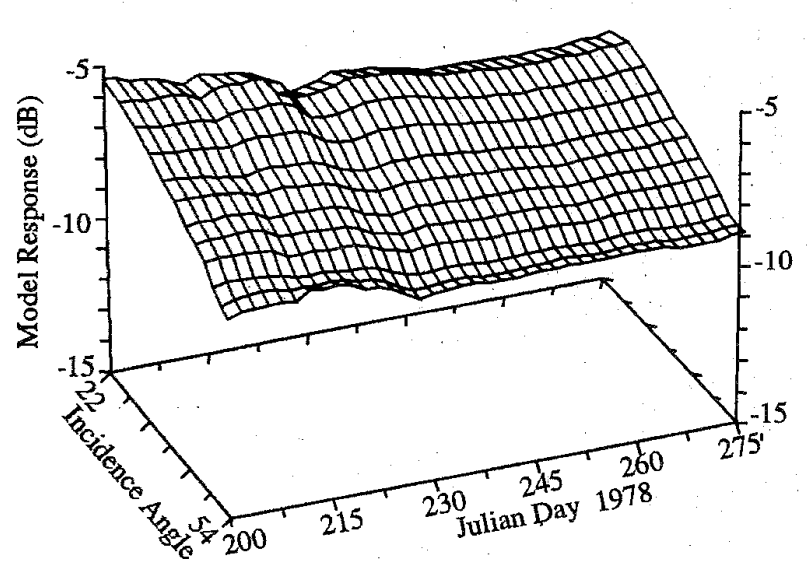

(b)

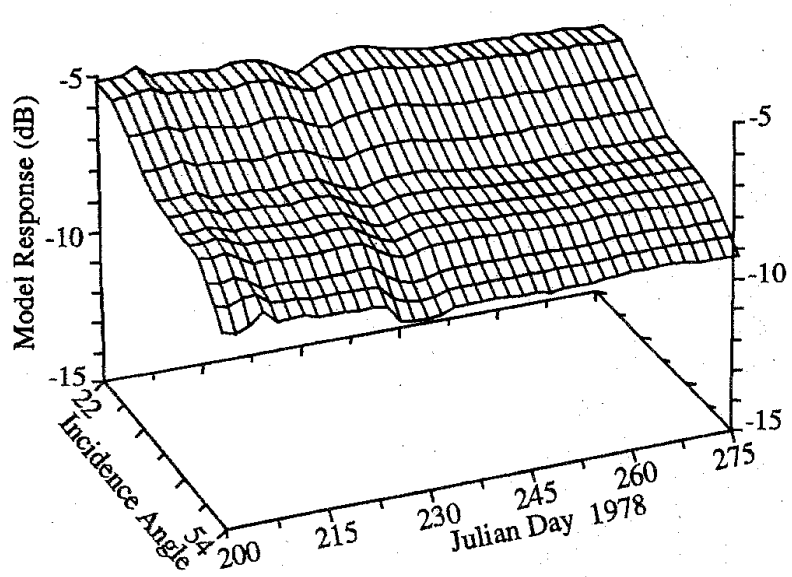

(d)

Fig. 10. Plots of the SASS model response $\left[\bar{F}\left(b_{n}, \theta\right)\right]$ versus incidence angle as a function of time. (a) Antenna 1. (b) Antenna 2. (c) Antenna 3. (d) Antenna 4. Note that the span of incidence angles for the measurements of each antenna differs, resulting in different incidence angle scales.

A plot of the temporal variation in $\mathcal{A}$ for the Escat data within the data mask is shown in Fig. 6. Comparison of this plot with Fig. 5 suggests that Escat data has much less temporal variation than does the SASS data with the beams balanced to within approximately $\pm 0.1 \mathrm{~dB}$ for a given pass, though, as in the SASS case a small difference $(0.25 \mathrm{~dB})$ in the $\mathcal{A}$ response for ascending and descending passes. The consistency also seen for the high-order coefficients suggesting that Escat data is remarkably consistent with relatively little temporal variation over the Amazon.

As before, the one $\sigma$ regression error for the full corrected data set provides an estimate of the average total $K_{P}$. Using the radiometric $K_{P}$ reported with the Escat data set (which corresponds to the rms of the $K_{P c}$ and $K_{P r}$ ), the residual $K_{P}$ is approximately $0.15 \mathrm{~dB}$, corresponding to the C-band $K_{P m}$ of the Amazon forest. Since this value is larger than the calibration corrections (less than $0.1 \mathrm{~dB}$ ) suggested by using our method, we conclude that Escat data is quite well calibrated and no corrections are required. The fact that the
Escat data exhibits less scatter may be due, in part, to the higher signal-to-noise ratio of the measurements (Escat has a much larger transmitter) and that the Escat measurements are processed through a single channel rather than the multiple channels used by SASS.

\section{CONCLUSION}

In this preliminary paper we have presented a very simple methodology for the relative calibration of a spaceborne scatterometer and have demonstrated the technique using SASS and Escat scatterometer data. Our technique is able to determine the beam-to-beam calibrations required to ensure consistency between the $\sigma^{\circ}$ measurements made by different antenna beams and correct for variations in processing channel gain. Corrections for time variation of the mean rain forest response can also be made. Unfortunately, our technique is limited by the inability to uniquely distinguish between seasonal variations in the backscatter of the rain forest and slow variations in the system gain. The residual temporal and 


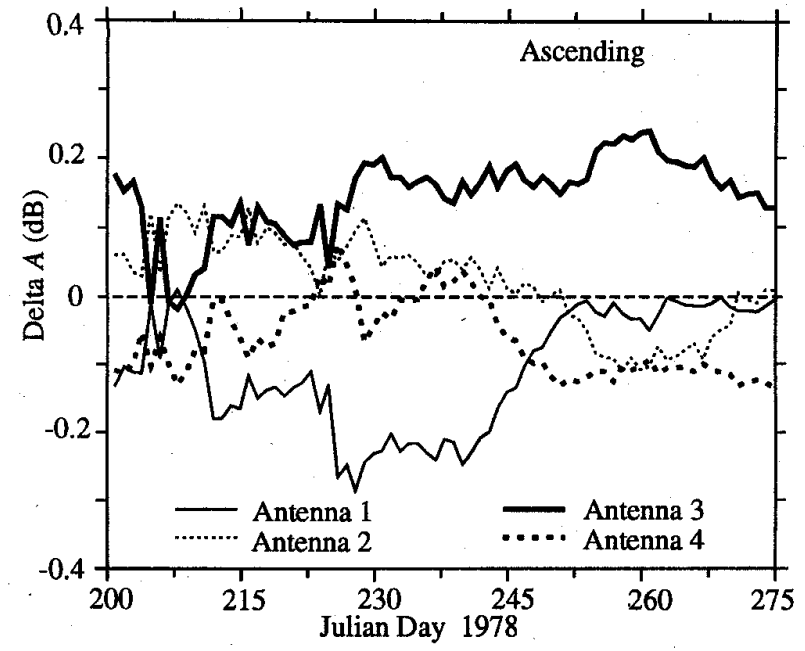

(a)

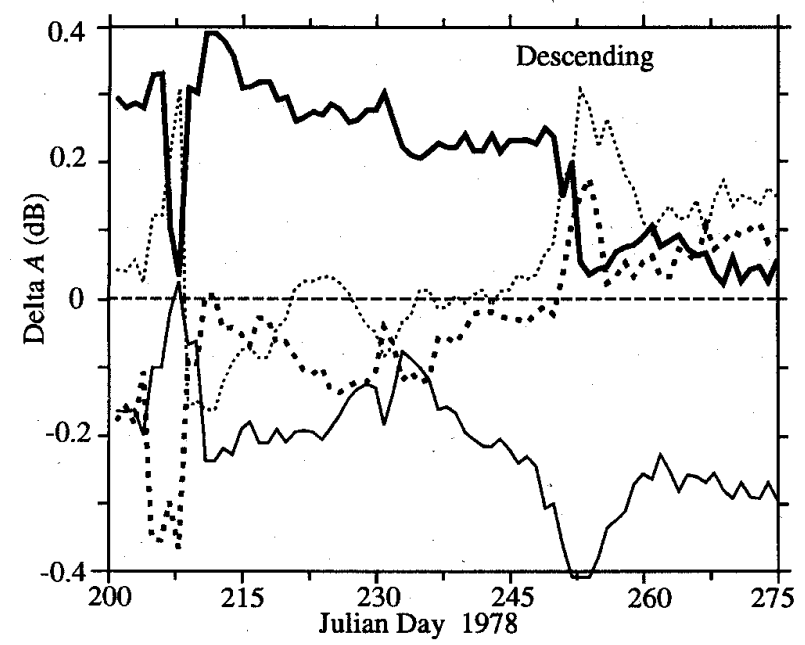

(b)

Fig. 11. Plot of the SASS $\mathcal{A}$ correction as a function of time.

spatial variability of the $\sigma^{\circ}$ of the rain forest is estimated to be $\pm 0.15 \mathrm{~dB}$ which represents the limit of the calibration accuracy possible with the Amazon rain forest. Using our technique we have found Escat scatterometer data to be much more stable than SASS data. SASS data exhibits significant temporal change (gain offset as large as $0.4 \mathrm{~dB}$ ) and a correction is suggested if a consistent, temporally stable result is desired. The postrecalibration error for SASS is estimated to be \pm 0.15 $\mathrm{dB}$ with a random distribution. Escat scatterometer data does not appear to need additional calibration correction. Over the Amazon the Escat data calibration is estimated to be within $\pm 0.1 \mathrm{~dB}$.

\section{ACKNOWLEDGMENT}

The authors would like to acknowledge the assistance of W. Davis in processing the Escat data. The SASS GDR and Escat data was obtained from the JPL PO.DAAC. The authors would like to thank ESA for the use of the Escat data.

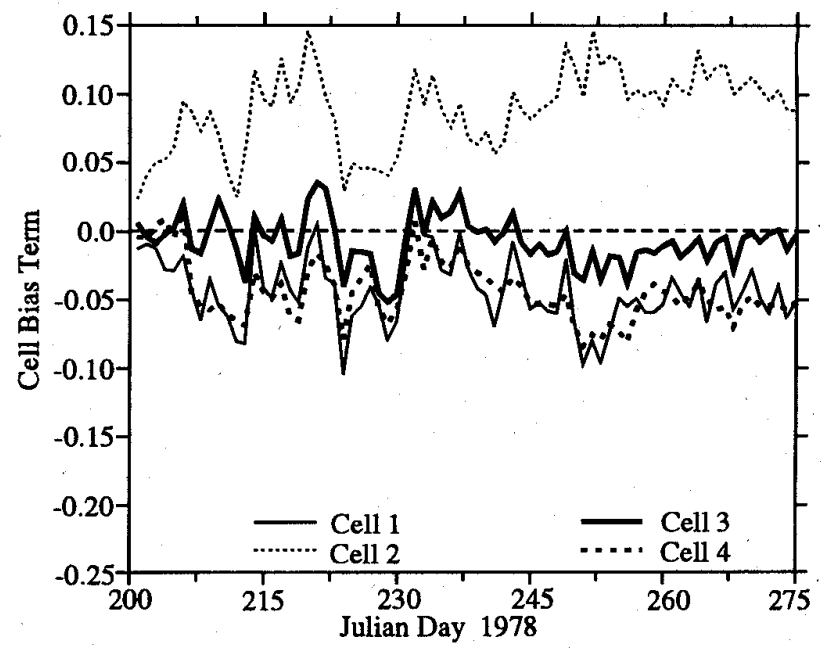

(a)

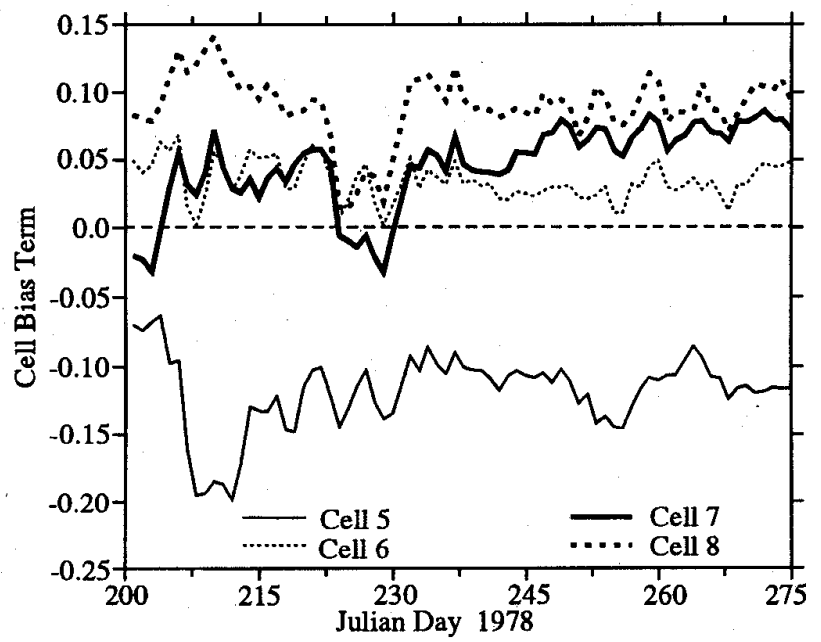

(b)

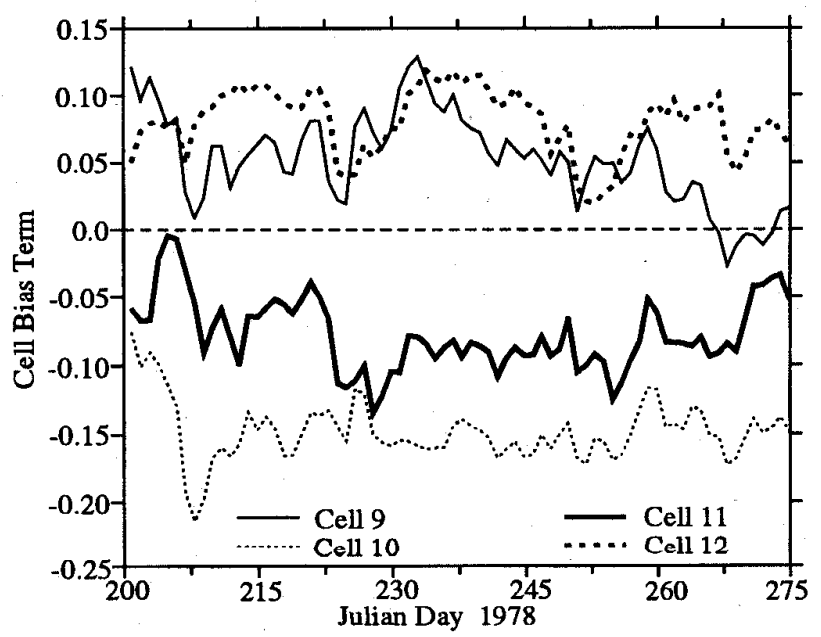

(c)

Fig. 12. Cell gain corrections for each of the 12 SASS channels as a function of time. 


\section{REFERENCES}

[1] I. J. Birrer, E. M. Bracalente, G. J. Dome, J. Sweet, and G. Berthold, " $\sigma$ signature of the Amazon rainforest obtained from the Seasat scatterometer," IEEE Trans. Geosci. Remote Sensing, vol. GRS-20, no. 1, pp. $11-17,1982$

[2] D. R. Daum, D. G. Long, and W. B. Davis, "Reconstruction enhanced resolution images from spaceborne microwave sensors," in Proc. Int. Geoscience and Remote Sensing Symp., Pasadena, CA, Aug. 8-12, 1994, pp. 2231-2233.

[3] R. G. Kennett and F. K. Li, "Seasat over-land scatterometer data, Part II: Selection of extended area land-target sites for the calibration of spaceborne scatterometers," IEEE Trans. Geosci. Remote Sensing, vol. 27, no. 6, pp. 779-788, Nov. 1989.

[4] D. G. Long, D. S. Early, and M. R. Drinkwater, "Enhanced resolution ERS-1 scatterometer imaging of southern hemisphere polar ice," in Proc. Int. Geoscience and Remote Sensing Symp., Pasadena, CA, Aug. 8-12, 1994, pp. 156-158.

[5] D. G. Long, P. Hardin, and P. Whiting, "Resolution enhancement of spaceborne scatterometer data," IEEE Trans. Geosci. Remote Sensing, vol. 31, no. 3, pp. 700-715, May 1993.

[6] D. G. Long and P. Hardin, "Vegetation studies of the Amazon Basin using enhanced resolution Seasat scatterometer data," IEEE Trans. Geosci. Remote Sensing, vol. 32, no. 2, pp. 449-460, Mar. 1994.

[7] F. Naderi, M. H. Freilich, and D. G. Long, "Spaceborne radar measurement of wind velocity over the ocean-An overview of the NSCAT scatterometer system," Proc. IEEE, vol. 79, no. 6, pp. 850-866, June 1991.

[8] G. B. Skouson, "Calibration of the seasat scatterometer using tropical rainforest," Master's thesis, Brigham Young Univ., Provo, UT, Dec. 1992.

[9] D. G. Long and M. R. Drinkwater, "Greenland observed at high resolution by the Seasat-A scatterometer," J. Glaciology, vol. 32, no. 2, pp. $213-230,1994$.

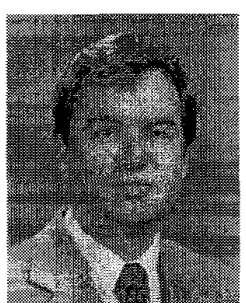

David G. Long (S'80-M'82) received the Ph.D. degree in electrical engineering from the University of Southern California, Los Angeles, in 1989.

From 1983 to 1990, he was with NASA's Jet Propulsion Laboratory where he developed advanced radar remote sensing systems. While at JPL, he was the Senior Project Engineer on the NASA Scatterometer. (NSCAT) project. NSCAT is planned for launch aboard the Japanese Advanced Earth Observing System (ADEOS) in August 1996. He is currently an Associate. Professor in the Electrical and Computer Engineering Department at Brigham Young University, where he teaches upper division and graduate courses in communications, microwave remote sensing, radar, and signal processing. $\mathrm{He}$ is the principle investigator on several NASA-sponsored interdisciplinary research projects in remote sensing including innovative radar systems, spaceborne scatterometry of the ocean and land, and modeling of atmospheric dynamics. He is a member of the NSCAT Science Working Team. He has numerous publications in signal processing and radar scatterometry. His research interests include microwave remote sensing, radar theory, spacebased sensing, estimation theory, computer graphics, signal processing, and mesoscale atmospheric dynamics.

Gary B. Skouson received the B.S. degree in electrical engineering and the M.S. degree in electrical engineering, both from Brigham Young University, Provo, UT, in 1991 and 1992, respectively.

While at BYU, he was a Teaching Assistant for the Electrical Engineering Department and a Research Assistant with the Microwave Earth Remote Sensing research group. Since 1992, he has been with IBM, Systems Technology and Architecture Division, Rochester, $\mathrm{MN}$, as an Engineer. 